\title{
Calibration of a Cross-Resonance Two-Qubit Gate Between Directly Coupled Transmons
}

\author{
A.D. Patterson, ${ }^{\dagger}$ J. Rahamim, T. Tsunoda, P.A. Spring, S. Jebari, K. Ratter, M. Mergenthaler, \\ G. Tancredi, B. Vlastakis, M. Esposito, and P.J. Leek ${ }^{*}$ \\ Department of Physics, Clarendon Laboratory, University of Oxford, Oxford OX1 3PU, United Kingdom
}

(Received 15 May 2019; revised manuscript received 7 September 2019; published 5 December 2019)

Quantum computation requires the precise control of the evolution of a quantum system, typically through application of discrete quantum-logic gates on a set of qubits. Here, we use the cross-resonance interaction to implement a gate between two superconducting transmon qubits with a direct static dispersive coupling. We demonstrate a practical calibration procedure for the optimization of the gate, combining continuous and repeated-gate Hamiltonian tomography with stepwise reduction of dominant two-qubit coherent errors through mapping to microwave control parameters. We show experimentally that this procedure can enable a $\hat{Z X}{ }_{-\pi / 2}$ gate with a fidelity $F=97.0(7) \%$, measured with interleaved randomized benchmarking. We show this in an architecture with out-of-plane control and readout that is readily extensible to larger-scale quantum circuits.

DOI: 10.1103/PhysRevApplied.12.064013

\section{INTRODUCTION}

A variety of hardware platforms are currently under intense development toward the realization of useful quantum computers, with several reaching a maturity at which moderate-fidelity quantum control can now be routinely achieved in few-qubit systems [1-5]. A key requirement for scaling such platforms to a practically useful level of quantum computation is the precise calibration of twoqubit quantum-logic gates to high fidelity, in an architecture that is practically scalable to many qubits $[6,7]$. This applies both for the development of useful imperfect near-term devices [8] as well for the pursuit of fully fault-tolerant error-corrected universal machines $[9,10]$. In the superconducting circuit platform, a large variety of methods for implementing two-qubit quantum logic have been proposed and demonstrated [11], in all cases using precisely shaped analog microwave pulses to deliver the control via microwave transmission lines. Coherent errors associated with imperfections in this microwave control can be mitigated through precise calibration of the analog control pulse parameters.

\footnotetext{
*peter.leek@physics.ox.ac.uk

†apatterson@oxfordquantumcircuits.com; now at Oxford Quantum Circuits, King Charles House, Oxford OX1 1JD, United Kingdom.

Published by the American Physical Society under the terms of the Creative Commons Attribution 4.0 International license. Further distribution of this work must maintain attribution to the author(s) and the published article's title, journal citation, and DOI.
}

In a superconducting circuit architecture employing fixed-frequency statically dispersively coupled qubits, the microwave-activated cross-resonance interaction [12] can be used to implement two-qubit entangling operations $[13,14]$. The interaction is enabled by the application of a cross-resonant drive tone; a qubit drive that is resonant with the transition of a neighboring qubit. The hybridization of the two transmon qubits due to their dispersive coupling allows a local drive on one qubit that is resonant with the other, to introduce an interaction between them with a rate proportional to the amplitude of this drive [15]. As this tone can be applied to the device using the same control wiring as for single-qubit operations, cross resonance allows two-qubit gate implementation with little adaptation to processor design or control circuitry. This interaction has been successfully implemented through mutual coupling to an interaction-mediating resonator [13], allowing for the qubits to be well spatially separated, which in principle keeps crosstalk between the qubits and their associated control wiring low. In practice, control errors due to imperfections in the control lines as well as crosstalk between them will inevitably remain and this problem will be exacerbated by physical crowding as attempts are made to further scale two-dimensional (2D) designs.

In this paper, a systematic calibration procedure to reduce coherent control error in an all-microwave twoqubit gate is outlined in detail. This approach allows the reduction of unwanted single-qubit and two-qubit rotations that arise due to large crosstalk and transient behavior of the control lines, which may, for example, be caused by line dispersion or reflections at imperfectly matched interfaces in the control wiring. We show that 
in a readily extensible three-dimensionally (3D) integrated circuit architecture [16] with a direct capacitive coupling between coaxial transmons, one can employ the procedure to perform two-qubit quantum logic. This scenario is markedly different from that employing a mediating resonator for the coupling between qubits [13], since the control-line crosstalk easily dominates the desired crossresonance interaction. The systematic approach to calibration presented here is likely to be applicable broadly in two-qubit quantum control, regardless of the platform.

The procedure is divided into two major parts. First, a calibration of the applied microwave tones is carried out, using the Hamiltonian-tomography technique presented in Ref. [14]. This is used to estimate crosstalkcancellation parameters without concern for potential transient errors. Second, the minimization of the transient errors is addressed specifically using a tomographic technique based on repetition of the gate, which we call "repeated-gate tomography." We map the dominant two-qubit coherent errors to specific microwave control parameters using spin echoes. The entire procedure is validated on a device consisting of two off-resonant, statically coupled coaxial transmons, enabling a (CNOT-equivalent) $\hat{Z X}_{-\pi / 2}$ gate to be performed with a fidelity of $97.0(7) \%$.

\section{EXPERIMENTAL SETUP}

A sketch of the experimental device is shown in Fig. 1(a). It is a two-qubit version of the coaxial circuit quantum electrodynamics (QED) architecture presented in Ref. [16]. Coaxially shaped transmon qubits ("coaxmons") lying in one plane on the upper surface of a substrate are capacitively coupled to individual readout resonators on the lower surface of the substrate. Coaxial drive lines for control of both the transmon and resonator are brought in through the sample holder perpendicular to the substrate surfaces, capacitively coupling to these quantum components. Each qubit, readout resonator, and the associated control wiring is confined to a cylindrical volume with its axis running perpendicular to the substrate surface, producing an architecture that can be extended to larger 2D grids of qubits.

The cross-resonance gate is implemented in this architecture through the addition of a small static coupling $J$ between the transmons, achieved here by adding a capacitor between their outer electrodes. We choose this simple direct capacitive coupling instead of employing a mediating resonator in order to minimize the complexity and the number of degrees of freedom in our quantum circuit. Although this may initially appear likely to present a crosstalk challenge due to the proximity of the qubits, we show here that this can be effectively eliminated through good calibration.

Driving one qubit (Q1) at the first transition frequency of the other qubit (Q2) activates an interaction between them that takes place with a rate proportional to the amplitude, $\Omega_{12}$, of this cross-resonant drive [12]. However, other undesired effects are also caused by the presence of this drive. First, the "control" qubit (Q1) under direct drive will be driven off resonantly. Second, due to the presence of higher levels of the transmon, the "target" qubit (Q2) will be directly driven on resonance, an effect that will be referred to in this paper as quantum crosstalk. Third, any stray direct coupling of the control line of the control qubit (P1) to the target qubit (Q2) will cause additional on-resonant driving, an effect referred to here as classical crosstalk. Finally, there will be an always-on twoqubit cross-Kerr interaction as the result of the fixed static dispersive coupling, an effect that is ideally minimized by the choice of the circuit parameters (specifically, the (a)

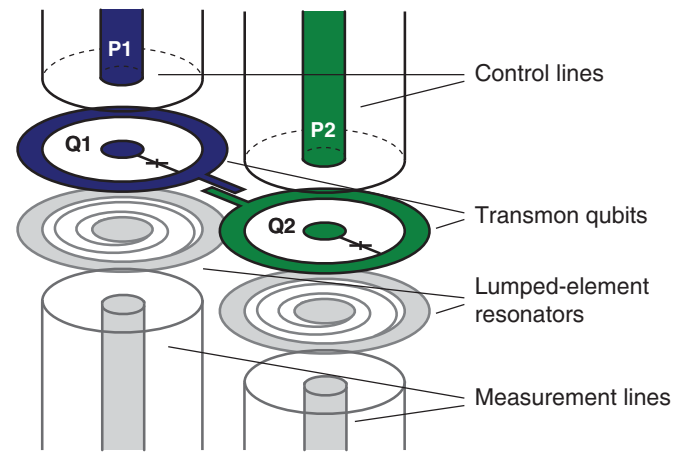

(b)

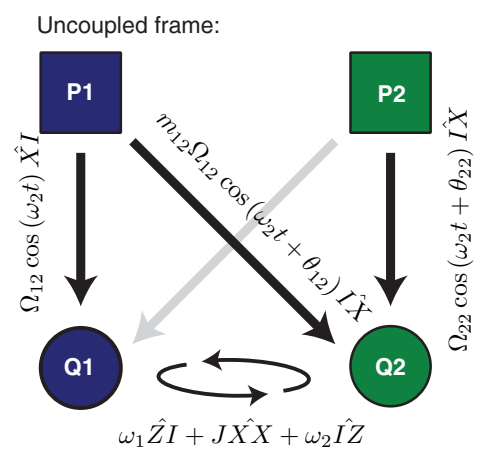

(c)

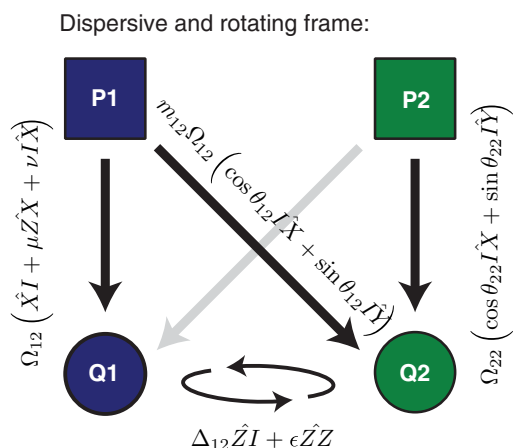

FIG. 1. A schematic of the device and the Hamiltonian. (a) A sketch of the coupled two-coaxial-transmon device with associated control lines and readout resonators. (b) A schematic of the full Hamiltonian with drive terms due to application of a cross-resonant drive to Q1 at the transition frequency of Q2 $(\mathrm{P} 1 \rightarrow \mathrm{Q} 1)$, a reduced direct application of this drive applied to Q2 due to classical crosstalk with relative amplitude $m_{12}$ and phase $\theta_{12}(\mathrm{P} 1 \rightarrow \mathrm{Q} 2)$, and an on-resonant direct correction drive applied directly to Q2 $(\mathrm{P} 2 \rightarrow \mathrm{Q} 2)$. (c) The transformation of the Hamiltonian terms as in (b) into the frame doubly rotating with the drives, in the dispersive low-power limit, $J, \Omega_{12} \ll \Delta_{12}$. 
detuning of the qubit transitions and the control qubits' anharmonicity).

The full Hamiltonian of the system, including drive terms, is shown pictorially in Fig. 1(b), where $m_{12}$ and $\theta_{12}$ are the relative amplitude and phase of the classical crosstalk term relative to the cross-resonance drive and $\Omega_{22}$ and $\theta_{22}$ are the amplitude and phase of a direct correction drive from port $\mathrm{P} 2$ to qubit $\mathrm{Q} 2$ (the cancellation tone).

In the frame in which both qubits rotate along with the drive and in the limit where $J, \Omega_{12} \ll \Delta_{12}$ (where $\Delta_{12}=$ $\omega_{1}-\omega_{2}$ is the detuning between the two transmons), the effective Hamiltonian takes the following form:

$$
\begin{aligned}
2 \hat{\mathcal{H}} / \hbar= & \Omega_{Z X} \hat{Z X}+\Omega_{Z Y} \hat{Z Y}+\Omega_{I X} \hat{I X}+\Omega_{I Y} \hat{I Y} \\
& +\Delta_{12} \hat{Z I}+\Omega_{X I} \hat{X I}+\Omega_{Y I} \hat{Y I}+\epsilon \hat{Z Z}
\end{aligned}
$$

where $\hat{Z X}=\hat{\sigma}_{z} \otimes \hat{\sigma}_{x}$, etc. The relevant contributions to each of the rates $\Omega$ are shown in the sketch in Fig. 1(c). In the figure, $\mu$ is the cross-resonance factor, $v$ is the quantum crosstalk factor, and $\epsilon$ is the cross-Kerr interaction factor.

The off-resonant control qubit drive terms are defined as $\Omega_{X I}=\Omega_{12} \cos \left(\theta_{12}\right)$ and $\Omega_{Y I}=\Omega_{12} \sin \theta_{12}$. They can be neglected when the drive amplitude $\Omega_{12} \ll \Delta_{12}$.

The two-qubit cross-resonance interaction terms $\Omega_{Z X}=$ $\mu \Omega_{12} \cos \theta_{12}$ and $\Omega_{Z Y}=\mu \Omega_{12} \sin \theta_{12}$ are similarly dependent on the cross-resonance drive amplitude and phase but also on the cross-resonance drive factor $\mu$. The single-qubit rotations on the target $\Omega_{I X}=\Omega_{12} v \cos \theta_{12}+$ $\Omega_{12} m_{12} \cos \left(\theta_{12}+\phi\right)$ and $\Omega_{I Y}=\Omega_{12} v \sin \theta_{12}+\Omega_{12} m_{12}$ $\sin \left(\theta_{12}+\phi\right)$ are given by the sum of the contribution from the quantum crosstalk characterized by the factor $v$ and the classical crosstalk characterized by the factor $m_{12}$ and relative phase $\theta_{12}$.

The terms $\epsilon, \mu$, and $v$ can be derived starting with the uncoupled Hamiltonian of the two transmons, finding the transformation that diagonalizes this Hamiltonian, and then transforming a drive on the control qubit in the initial nondiagonal Hamiltonian into the new diagonal frame. A detail of the theory behind a perturbative approach to this is presented in Refs. [15,17]. The resulting expressions considering the first three energy levels only of each transmon are as follows:

$$
\begin{aligned}
\mu & =-\frac{J}{\Delta_{12}} \frac{\alpha_{1}}{\Delta_{12}+\alpha_{1}}, \\
v & =-\frac{J}{\Delta_{12}} \frac{\Delta_{12}}{\Delta_{12}+\alpha_{1}}, \\
\epsilon & =J^{2} \frac{\alpha_{1}+\alpha_{2}}{\left(\Delta_{12}+\alpha_{1}\right)\left(\Delta_{12}-\alpha_{2}\right)} .
\end{aligned}
$$

Table I shows the relevant parameters of the two-qubit device used in our experiments.

\section{CROSSTALK CANCELLATION}

In this section, we describe the first part of the calibration process, which is used to initially reduce coherent error due to crosstalk. From finite-element electromagnetic simulations of the device, we expect to have a classical crosstalk factor $m_{12}$ of order $5 \%$. Since this is larger than $\mu=2.4 \%$ (calculated using Eq. (2a) and Table I), it is itself sufficient to cause the single-qubit rotation terms $\Omega_{I X}$ and $\Omega_{I Y}$ to dominate the dynamics of the system, without considering the level of quantum crosstalk. The use of echoing schemes alone to reduce this error is not possible, as these terms may be larger than or of a similar order to the cross-resonance terms and will not commute with them in general. In order to reduce the $\Omega_{I X}$ and $\Omega_{I Y}$ terms, we introduce a cancellation tone directly applied to the target qubit at its own transition frequency with amplitude $\Omega_{22}$ and phase $\theta_{22}$.

The optimal values for the amplitude and phase of the cancellation tone are obtained using a procedure similar to that presented in Ref. [14]. First, a cross-resonant microwave pulse of fixed amplitude and varying length $t$ is applied to the control qubit. Using single-qubit tomography measurements on both qubits, the dynamics of the two individual qubits in time are recorded. The results for the target qubit are fitted to a fixed-axis fixed-frequency rotation about the Bloch sphere. This is possible since the control qubit begins (approximately) in state $|0\rangle$, an eigenstate of $\hat{Z I}$. This operator commutes with all terms of the Hamiltonian except $\hat{X I}, \hat{Y} I$, which we can safely neglect since $\Omega_{X I, Y I} \ll \Delta_{12}$. The dynamics are therefore reduced to the single-qubit dynamics of the target. Repetition of the process with the control qubit initially prepared in state |1) allows $\Omega_{Z X}, \Omega_{Z Y}, \Omega_{I X}$, and $\Omega_{I Y}$ to be deduced. $\Omega_{I Z}$ and $\Omega_{Z Z}$ can also be measured, in theory returning the values 0

\begin{tabular}{|c|c|c|c|}
\hline Qubit & & Q1 & Q2 \\
\hline Transition frequency & $\omega / 2 \pi(\mathrm{GHz})$ & 6.509 & 5.963 \\
\hline Anharmonicity & $\alpha / 2 \pi(\mathrm{MHz})$ & -300 & -314 \\
\hline Relaxation time & $T_{1}(\mu \mathrm{s})$ & 16.2 & 23.9 \\
\hline Coherence time & $T_{2}(\mu \mathrm{s})$ & 25.1 & 35.2 \\
\hline Pure dephasing time & $T_{\phi}(\mu \mathrm{s})$ & 111 & 134 \\
\hline $\begin{array}{l}\text { One-qubit (1Q) } \\
\text { Clifford-gate } \\
\text { fidelity }\end{array}$ & $F_{1 Q}$ & $99.4 \%$ & $99.2 \%$ \\
\hline Cross-Kerr shift & $\Omega_{Z Z} / 2 \pi(\mathrm{MHz})$ & \multicolumn{2}{|c|}{-0.33} \\
\hline Qubit-qubit coupling & $J / 2 \pi(\mathrm{MHz})$ & \multicolumn{2}{|c|}{10.7} \\
\hline $\begin{array}{l}\text { Cross-resonance } \\
\text { factor }\end{array}$ & $\mu$ & \multicolumn{2}{|c|}{2.4} \\
\hline $\begin{array}{l}\text { Quantum-crosstalk } \\
\text { factor }\end{array}$ & $v$ & \multicolumn{2}{|c|}{4.3} \\
\hline
\end{tabular}
and $\epsilon$ as predicted.

TABLE I. A summary of the device parameters: the relevant parameters of the statically coupled two-coaxial-transmon device used to test the calibration procedure. 
The cancellation tone can then be introduced to the system with a chosen amplitude $\Omega_{22}$ and phase $\theta_{22}$ such that its additive contributions to $\Omega_{I X}$ and $\Omega_{I Y}$ are the negative of the values measured previously. In practice, an iterative procedure can be used to find these parameters quickly [18]. Such a process may be useful if nonlinearities in the cross-resonance interaction need to be corrected [19].

Figure 2(b) shows the initial measurement in this process with no cancellation tone applied and Fig. 2(c) shows a final measurement with the properly calibrated cancellation tone applied. The cross-resonant drive phase and amplitude are changed during the procedure to target $\Omega_{Z Y}=0$ and $\Omega_{Z X}=\Omega_{\mathrm{CR}}^{\text {trgt }}$, where $\Omega_{\mathrm{CR}}^{\mathrm{trg} t}$ is a selected target rate at which to drive the cross-resonance interaction. A sketch of the pulse scheme used is shown in Fig. 2(d), while a sketch of the iterative procedure used for the minimization of the crosstalk errors is shown in Fig. 2(e).

From the cross-resonance interaction rate and the estimated values $\mu=2.4 \%$ and $v=4.3 \%$, one can calculate the expected contribution of quantum crosstalk to $\Omega_{I X}$ and $\Omega_{I Y}$, and subtract it from the total crosstalk. The remaining classical crosstalk must be the direct result of the drive. This gives the value of the classical-crosstalk parameter $m_{12}=7.1 \%$ (corresponding to an isolation of approximately $-23 \mathrm{~dB})$.

\section{A. Echo-gate pulse scheme}

Using the results shown in Fig. 2(c), an effective Hamiltonian of the form

$$
2 \hat{\mathcal{H}} / \hbar \approx \Omega_{\mathrm{CR}}^{\mathrm{trgt}} \hat{Z X}+\epsilon \hat{Z Z}
$$

can be realized.

If $\Omega_{\mathrm{CR}}^{\text {trgt }}$ is much larger than $\epsilon$ and any calibration errors, one may expect a pulse of length $t=\Omega_{\mathrm{CR}}^{\text {trgt }} / 8 \pi$ to perform a good approximation of a $Z \hat{X}_{-\pi / 2}$ gate. In practice, $\epsilon$ is often non-negligible and small errors in the calibration of the cancellation tone can be present (due to inaccurate calibration or parameter drift).

One can reduce these errors in a manner that is robust to slight parameter drift using the echo-gate scheme presented in Ref. [14]. This involves performing the crossresonance gate of length $t$ in two components of length $t / 2$, negating the amplitude of the second component, and preceding and following it with an $\hat{X I}_{\pi}$ single-qubit gate (a)

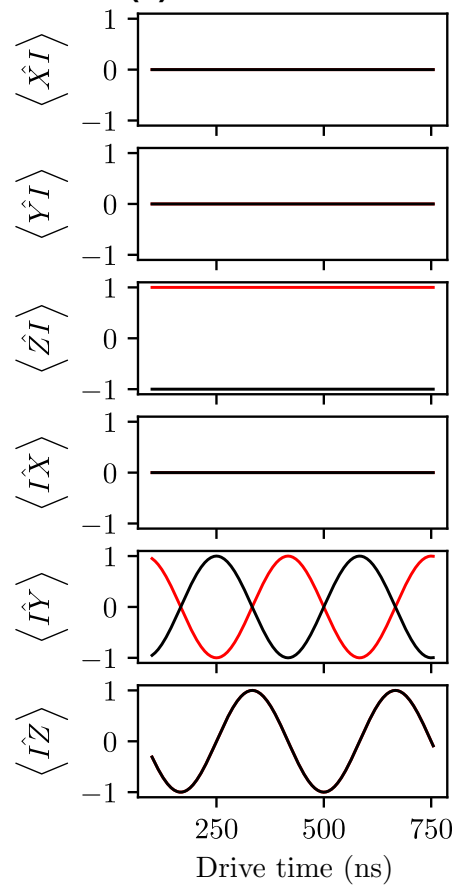

(b)
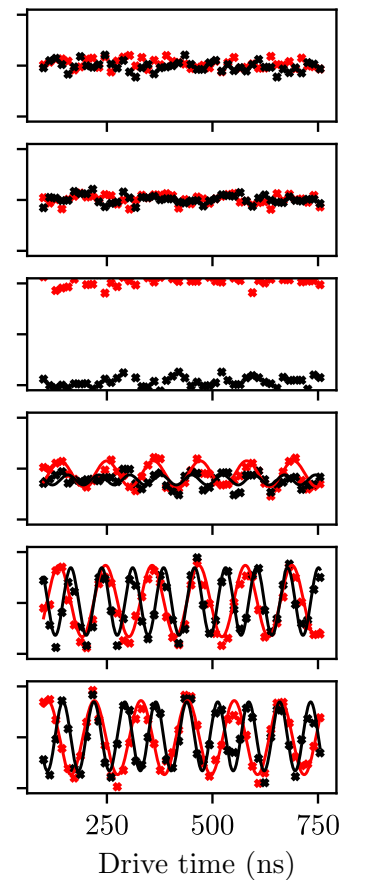

(c)
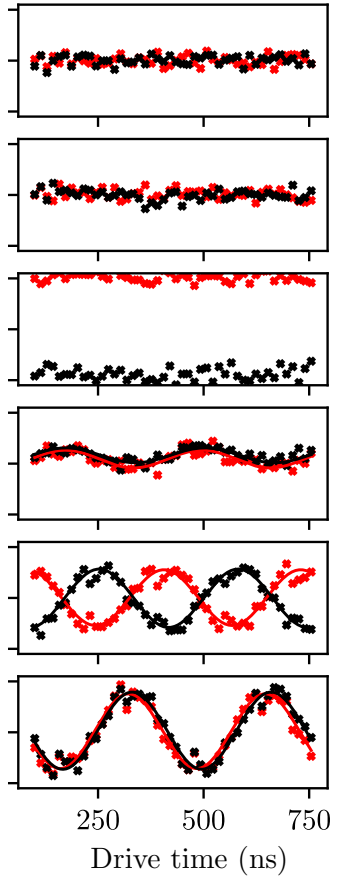

(d)

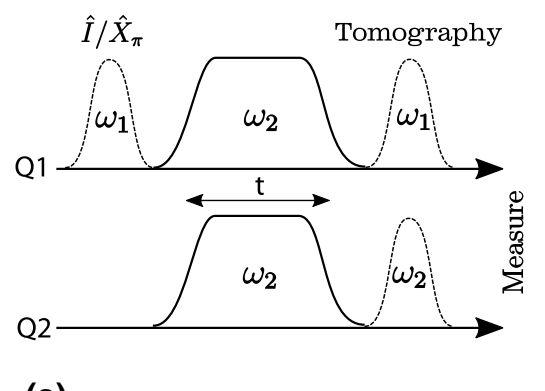

(e)

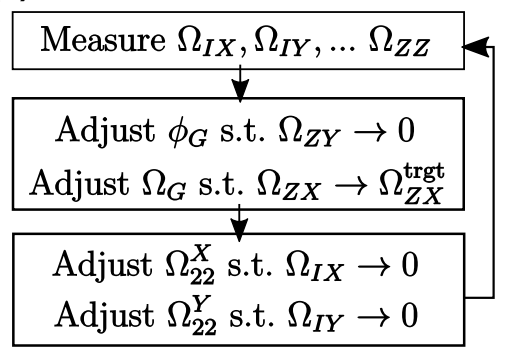

FIG. 2. Continuous-wave cross-resonance tuneup with crosstalk cancellation: the results of simultaneous single-qubit tomography on both qubits after a variable length cross-resonant drive pulse has been applied to Q1. For the data shown in red (black), Q1 is initially prepared in state $|0\rangle(|1\rangle)$. (a) The expected results for a Hamiltonian of the form $\mathcal{H} / \hbar=\Omega_{Z X} / 2 Z \hat{Z X}$ with $\Omega_{Z X} / 2 \pi=3.0 \mathrm{MHz}$. (b) The results using an initial estimate of the cross-resonant drive amplitude to achieve this Hamiltonian without the application of a cancellation tone. (c) The results after the amplitude and phase of the cross-resonance tone as well as an introduced cancellation tone are adjusted to give as close to the desired Hamiltonian as possible. (d) A sketch of the pulse scheme used in experiments shown in (b) and (c). The pulse shape used for the cross-resonance tone is a flat top with hyperbolic tangent (characteristic time $10 \mathrm{ns)} \mathrm{rise} \mathrm{and} \mathrm{fall.}$ (e) An outline of the iterative procedure used to reach the final results shown in (c). 


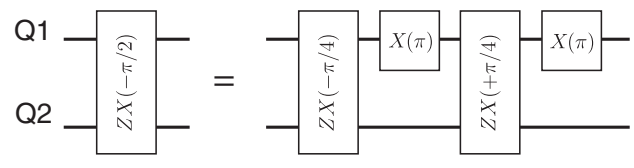

FIG. 3. The echo-gate scheme. A $\hat{Z X}_{-\pi / 2}$ gate can be formed of two $\hat{X}_{\pi}$ gates on the control qubit, a $Z \hat{X}_{-\pi / 4}$ and a $Z \hat{X}_{+\pi / 4}$ gate. This gate scheme makes the $Z \hat{X}_{-\pi / 2}$ gate more resilient to coherent errors common in the $\hat{Z X}_{ \pm \pi / 4}$ primitives [14].

applied to the control qubit. During the second component, the amplitudes of all terms proportional to the drive amplitude $\left(\Omega_{I X}, \Omega_{I Y}, \Omega_{Z X}\right.$, and $\left.\Omega_{Z Y}\right)$ are negated, as are the terms proportional to the control $\hat{Z}$ operator $\left(\Omega_{Z X}, \Omega_{Z Y}\right.$, and $\left.\Omega_{Z Z}\right)$. The only terms not effectively cancelled are $\Omega_{Z X}$ and $\Omega_{Z Y}$, the cross-resonance interaction terms, as they are negated twice (see Fig. 3). The effective Hamiltonian across the entire time $t$ of the sequence is then as follows:

$$
2 \hat{H} / \hbar \approx \Omega_{\mathrm{CR}}^{\mathrm{trgt}} \hat{Z X} .
$$

This approximation is valid when all terms not commuting with $\hat{Z X}$ in the true Hamiltonian are zero or small in comparison to $\Omega_{Z X}$. This means that with large values of $m_{12}$ compared to $\mu$, the procedure for cancellation of crosstalk presented previously must be performed and a cancellation tone used before an echo scheme such as the one presented here will begin to help improve the gate quality. Note that if the largest remaining term in the Hamiltonian after the previous calibration is $\Omega_{Z Z}$, as expected, then this echo gate results in an effective Hamiltonian with a smaller error, in which the leading-order term will be proportional to $\hat{I Y}[18]$.

\section{TRANSIENT-ERROR REDUCTION}

In this section, we describe the second part of the calibration process, which corrects for transient errors using the technique of "repeated-gate tomography."

\section{A. Repeated-gate tomography}

All calibration measurements to this point are performed with pulses of continuously varied lengths and the data are modeled as a constant-rate rotation about a fixed axis. This means that all Hamiltonian estimates are based upon the extension of a fixed-amplitude segment of the pulse for a small time step. Transient coherent errors due to edge effects of the pulse (such as reflections at the sample-holder interface) during the rise and fall of the final gate may be present. If so, their contribution will have an effect on the final gate operation that has not yet been taken into account by the calibration procedure.

To alleviate this issue, we employ a technique that we call "repeated-gate tomography." Rather than extending the pulse length continuously in time, we instead discretize the pulse length into an integer number of gate repetitions and repetitions of the gate (including in some cases the entire echo scheme discussed previously) are performed in place of variable-length gates. Figure 4(a) shows a sketch of the pulse scheme used.

In Fig. 4(b), we show the results of single-qubit tomography on Q2 after a varying number of repetitions of the $Z X_{-\pi / 4}$ gate are applied to the two-qubit system and compare them with the ideal case (dashed lines). To find the effective Hamiltonian, we fit the fixed-axis fixed-rotationrate model to the data set with a relatively low sampling rate compared to the expected oscillation frequency. If the previous calibration measurements are accurate and the echo scheme is used, the frequency of oscillations in the resulting data will not prevent correct interpretation. Ascertaining the effective Hamiltonian values (now measured for convenience in units of cycles per gate rather than cycles per second) now allows coherent rotations caused by transient effects during the rise and fall of each pulse to be properly taken into account. In the example shown in Fig. 4, the $Z \hat{X}_{-\pi / 4}$ gate in the sequence is performed at a rate of $3 \mathrm{MHz}$, which - with the inclusion of $10 \mathrm{~ns}$ tanhshaped rise-and-fall times - results in a total gate time of $62 \mathrm{~ns}$. In this and all other experiments, the single-qubit
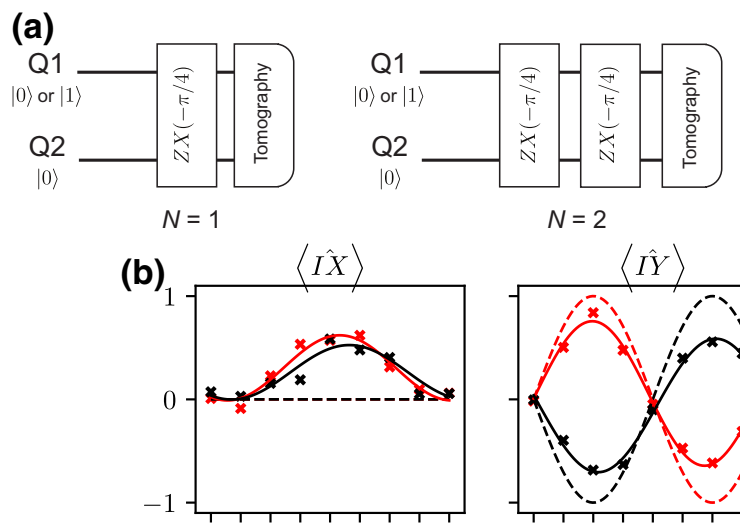

$\begin{array}{lllllllll}0 & 1 & 2 & 3 & 4 & 5 & 6 & 7 & 8\end{array}$

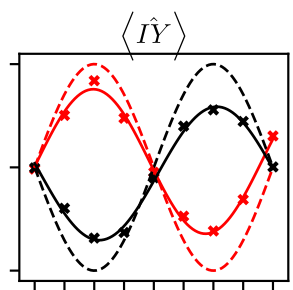

$\begin{array}{lllllllll}0 & 1 & 2 & 3 & 4 & 5 & 6 & 7 & 8\end{array}$ Gate Repetitions, N
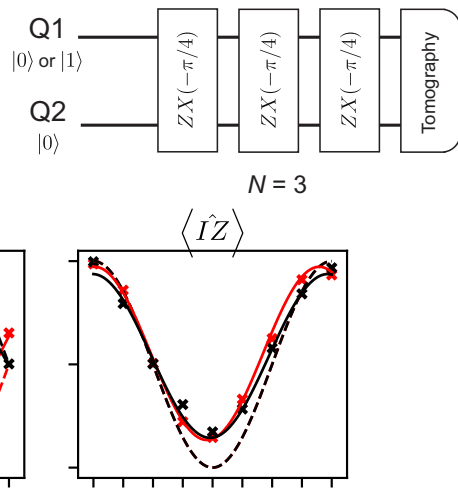

$\begin{array}{lllllllll}0 & 1 & 2 & 3 & 4 & 5 & 6 & 7 & 8\end{array}$

FIG. 4. Gate characterization with repeated-gate Hamiltonian tomography. (a) The pulse scheme used to characterize the $\hat{Z X}_{-\pi / 4}$ gate. (b) The single-qubit tomography results on Q2 after varying repetition numbers of the $\hat{Z X}_{-\pi / 4}$ gate are applied to the two-qubit system. The red (black) data are collected after Q1 is initially prepared in $|0\rangle(|1\rangle)$. The dashed lines show the ideal-case result for a $\hat{Z X}_{-\pi / 4}$ gate. 
gates are $40 \mathrm{~ns}$ in total length. This gives a total gate time for a $Z X_{-\pi / 2}$, incorporating the echo sequence, of 204 ns.

Figure 5 shows the extracted effective Hamiltonian from many repetitions of the experiment in Fig. 4 as various parameters of the gate are varied in a small range around the previous best estimate.

The parameters are varied in the following order. First, the global phase $\phi_{G}$ is adjusted, meaning that the phase of both the cross-resonant $\theta_{12}$ and cancellation $\theta_{22}$ drives are offset from their previous values, denoted by $\theta_{12}^{\prime}$ and $\theta_{22}^{\prime}$, respectively:

$$
\theta_{12}=\theta_{12}^{\prime}+\phi_{G}, \quad \theta_{22}=\theta_{22}^{\prime}+\phi_{G}
$$

$\Omega_{Z Y}$ has a sinusoidal dependence on $\phi_{G}$ and so if the previous estimate is good, then $\Omega_{Z Y}$ will be approximately linearly dependent on $\phi_{G}$. The estimate for the global-phase parameter is therefore improved by fitting the extracted $\Omega_{Z Y}$ values to a linear model and extracting the zero crossing point, as shown in the first inset in Fig. 5(a).

Second, the global amplitude $\Omega_{G}$ is adjusted. This is the adjustment of the amplitude of the cross-resonant $\Omega_{12}$ and cancellation $\Omega_{22}$ drive amplitudes about their previous values, $\Omega_{12}^{\prime}$ and $\Omega_{22}^{\prime}$, while keeping a fixed ratio between them:

$$
\Omega_{12}=\Omega_{G}+\Omega_{12}^{\prime}, \quad \Omega_{22}=\Omega_{G} \frac{\Omega_{22}^{\prime}}{\Omega_{12}^{\prime}}+\Omega_{22}^{\prime} .
$$

$\Omega_{Z X}$ has a theoretically linear dependence on $\Omega_{G}$ provided that $\Omega_{12}$ remains much less than $\Delta_{12}$. The estimate for the global-amplitude parameter is therefore improved by fitting the $\hat{Z X}$ rotation rate to a linear model and extracting the crossing point with a rotation rate of -0.25 cycles per gate, as shown in the second inset in Fig. 5(a).

The in-phase and quadrature-phase components of the cancellation tone $\Omega_{22}^{X}=\Omega_{22} \cos \theta_{22}$ and $\Omega_{22}^{Y}=\Omega_{22} \sin \theta_{12}$ are now sequentially adjusted, by individually offsetting them within a small fraction of the total cancellation-tone amplitude.

The measured $\Omega_{I X}$ and $\Omega_{I Y}$ Hamiltonian terms are then fitted to linear models in the two cases and the relevant parameter updated to the value of the zero-crossing point. In order to calibrate these parameters, the echo scheme must not be used, so that measured single-qubit rotations of the target qubit alter with the cancellation tone in a simple manner. During these calibration stages, therefore, a single microwave pulse of length $t / 2$ is applied to both the control and the target (an approximate $Z X_{-\pi / 4}$ gate) and is repeated a variable number of times. After calibration of
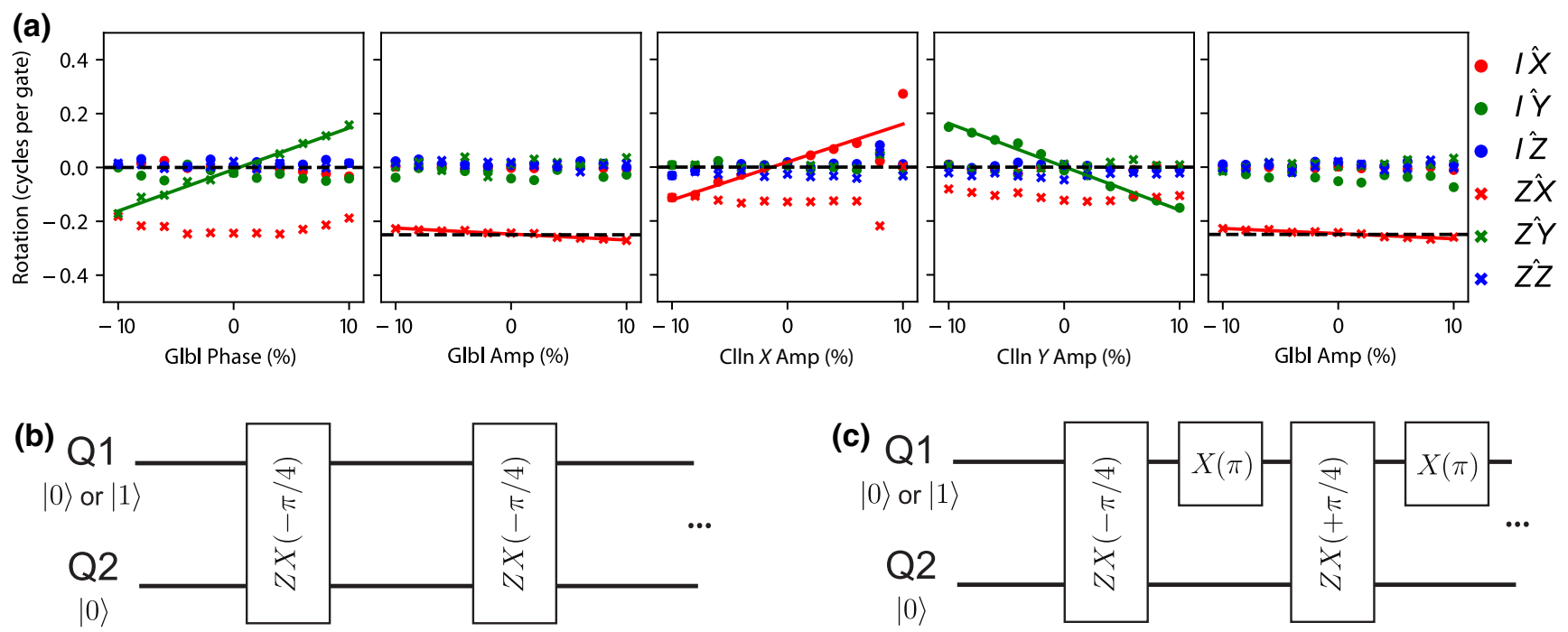

FIG. 5. The correction of transient errors. (a) Each subplot shows the results of the effective Hamiltonian fit to multiple iterations of an experiment as shown in Fig. 4(b) with total repetitions 0 through 8, as a physical parameter of the gate implementation is swept in a small range around the previous best estimate. The global ("Glbl") phase (the phase of both pulses with fixed offset) is first swept in a range to correct the $\hat{Z Y}$ term. The global amplitude (the amplitude of both pulses with a fixed ratio) is then swept to correct $\hat{Z X}$ to $-1 / 4$ cycles per gate. Next, the two quadratures of the cancellation tone ("Clln $X$ Amp" and "Clln $Y$ Amp") are swept individually to reduce the remaining $I X$ and $\hat{I Y}$ terms to zero, before a final recorrection of the $\hat{Z X}$ term. (b) The gate scheme used to deduce the effective Hamiltonian for each value of the Clln $X$ Amp and Clln $Y$ Amp parameters in (a). The echo scheme is not used here, as it reduces the error in $I X$ and $\hat{I Y}$ to which these parameters are fitted. (c) The gate scheme used to deduce the effective Hamiltonian for each value of the global-phase and global-amplification parameters. The echo scheme can be used here, as it does not affect the errors in $\hat{Z X}$ or $\hat{Z Y}$ to which these parameters are fit. 
these parameters, $\Omega_{G}$ is again adjusted to correct the total rotation angle after changes to the other parameters.

The adjustments made in the case shown in Fig. 5(a) appear slight, yet from the simple linear model we predict them to have increased the final gate fidelity by $1.1 \%$.

\section{B. Characterization and benchmarking}

Process tomography [20] and interleaved randomized benchmarking [21] are used to assess the fidelity $F$ of gates optimized using the procedure presented above (see Fig. 6). The entire procedure including benchmarking can be performed for different gate lengths to find the optimal value for $\Omega_{\mathrm{CR}}^{\mathrm{trgt}}$ and the results discussed below are for an optimal value for this particular device of $\Omega_{\mathrm{CR}}^{\mathrm{trgt}} / 2 \pi=$ 3.0 MHz.

We first characterize the $\hat{Z X}_{-\pi / 2}$ gate using quantum process tomography (QPT) [20]. We see from the tomogram in Fig. 6(a) that $Z X_{-\pi / 2}$ is the dominant term and find a gate-fidelity estimate of $F_{\mathrm{PT}}=99 \%$, using the method in Ref. [22]. We must be careful with this result, however, since state preparation, measurement, and leakage errors can affect QPT. Using noise resampling and maximum likelihood estimation, we find that the gate-fidelity estimate is bounded $F_{\mathrm{PT}}>91.6 \%$ within a $90 \%$ confidence interval. Numerical analysis of the tomogram also confirms the leading remaining coherent error to be in $\hat{I Y}$, as expected.

For a more accurate assessment of the gate fidelity, we carry out interleaved randomized benchmarking [21] [see Fig. 6(b)], a more reliable method not affected by statepreparation and measurement errors. We obtain a fidelity estimate of $F_{\text {IRB }}=97.0(7) \%$, with the error in a $90 \%$ confidence interval. Note that the estimated population of $|00\rangle$ should decay to 0.25 but is found to decay to approximately 0.19 in both the standard and the interleaved case. This can be attributed to imperfect measurement calibration or leakage of the system out of the computational subspace. We can calculate the impact of these scenarios on our benchmarking result. If we assume that the measurement calibration is perfect and attribute the $6 \%$ deficit purely to leakage, we estimate the fidelity of the gate to be $F \approx 96.7 \%$. If we instead assume a worst-case scenario that the system has completely left the computational subspace by the point of randomization, we estimate the fidelity to be $F \approx 95.9 \%$.

The predicted fidelity limit for a gate of this length is 98.9\% when taking into account both decoherence and single-qubit-gate error, an error rate approximately 2.7 times smaller than that seen here. There are two likely sources of this discrepancy. A remaining coherent singlequbit error $(\hat{I Y}$, resulting from the echoed-out $\hat{Z Z}$ interaction) could be corrected to further increase the fidelity. This cross-Kerr interaction could also be reduced in future designs. Leakage out of the computational subspace to higher transmon-energy levels may also contribute to the gate error.

Simple repetition of this procedure with different target cross-resonance rates in order to search for the optimum gate length can take several hours, largely due to the time required to perform randomized benchmarking. This problem could be mitigated by development of an error model to allow the optimum value of $\Omega_{\mathrm{CR}}^{\text {trgt }}$ to be predicted. Alternatively, full numerical optimization (a)

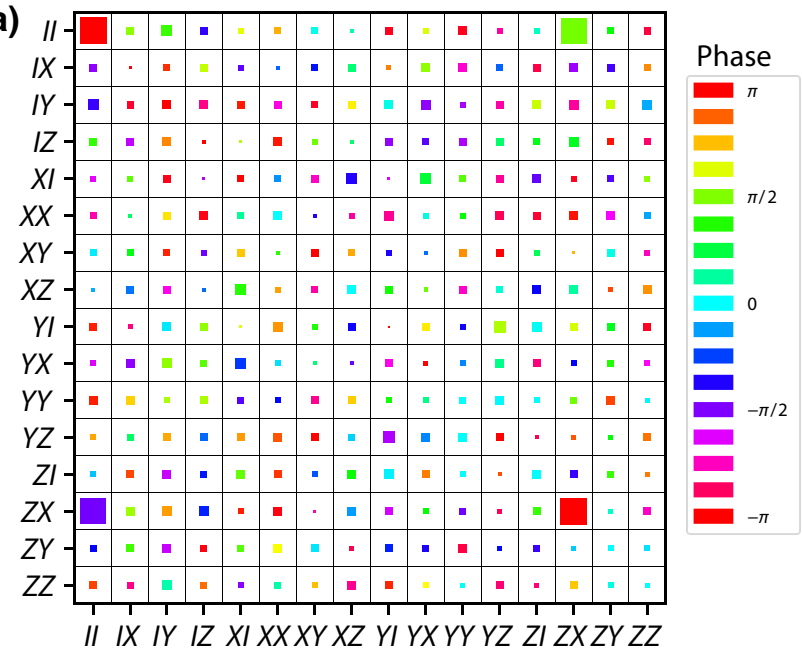

(b)

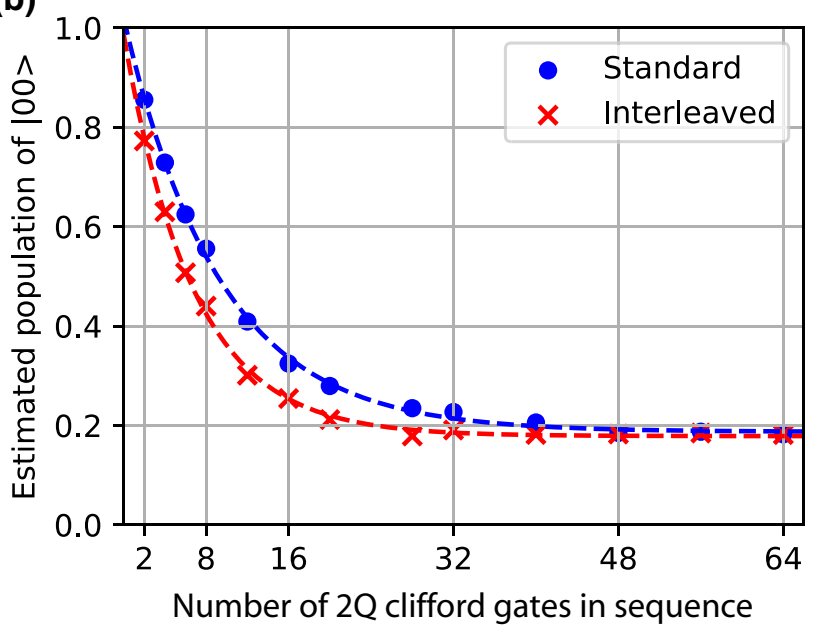

FIG. 6. Characterization and benchmarking of the optimized gate. The standard techniques of quantum process tomography and interleaved randomized benchmarking are performed on the optimized gate. (a) The process tomogram (the displayed matrix is $\chi$, the Kraus decomposition of the transfer matrix) provides qualitative confirmation that a $\hat{Z X}_{-\pi / 2}$ gate is applied to the system. (b) The results of interleaved randomized benchmarking show that the gate has a fidelity of $97.0 \% \pm 0.7 \%$. The error given is a $90 \%$ confidence interval. 
of the cross-resonance drive pulse could be considered $[23,24]$.

\section{CONCLUSION}

In conclusion, we present a method for the calibration of the cross-resonance two-qubit gate to reduce error caused by classical crosstalk and imperfectly matched control lines. The method is validated using a two-qubit implementation of coaxial circuit QED [16] with directly coupled transmons. The procedure outlined here is applicable to other physical systems that use a driven crossresonance two-qubit interaction and can serve as a general guide for mitigating unwanted coherent errors in quantum processors.

\section{ACKNOWLEDGMENTS}

This work has received funding from the United Kingdom Engineering and Physical Sciences Research Council under Grants No. EP/J013501/1, No. EP/M013243/1, and No. EP/N015118/1 and from Oxford Quantum Circuits Limited. A.P. acknowledges Oxford Instruments Nanoscience, M.M. acknowledges the Stiftung der Deutschen Wirtschaft (sdw), and T.T. acknowledges the Masason Foundation and the Nakajima Foundation for financial support.

[1] S. Debnath, N. M. Linke, C. Figgatt, K. A. Landsman, K. Wright, and C. Monroe, Demonstration of a small programmable quantum computer with atomic qubits, Nature 536, 63 (2016).

[2] Ruoyu Li, Luca Petit, David P. Franke, Juan Pablo Dehollain, Jonas Helsen, Mark Steudtner, Nicole K. Thomas, Zachary R. Yoscovits, Kanwal J. Singh, Stephanie Wehner, Lieven M. K. Vandersypen, James S. Clarke, and Menno Veldhorst, A crossbar network for silicon quantum dot qubits, Sci. Adv. 4, 3960 (2018).

[3] Andreas Reiserer, Norbert Kalb, Machiel S. Blok, Koen J. M. van Bemmelen, Tim H. Taminiau, Ronald Hanson, Daniel J. Twitchen, and Matthew Markham, Robust Quantum-Network Memory Using Decoherence-Protected Subspaces of Nuclear Spins, Phys. Rev. X 6, 021040 (2016).

[4] Xi-Lin Wang, Luo-Kan Chen, W. Li, H.-L. Huang, C. Liu, C. Chen, Y.-H. Luo, Z.-E. Su, D. Wu, Z.-D. Li, H. Lu, Y. Hu, X. Jiang, C.-Z. Peng, L. Li, N.-L. Liu, Yu-Ao Chen, and Chao-Yang Lu, Jian-Wei Pan, Experimental Ten-Photon Entanglement, Phys. Rev. Lett. 117, 210502 (2016).

[5] C. Neill et al., A blueprint for demonstrating quantum supremacy with superconducting qubits, Science 360, 195 (2018).

[6] E. Knill, Quantum computing with realistically noisy devices, Nature 434, 39 (2005).

[7] Austin G. Fowler, Matteo Mariantoni, John M. Martinis, and Andrew N. Cleland, Surface codes: Towards practical large-scale quantum computation, Phys. Rev. A 86, 032324 (2012).
[8] John Preskill, Quantum computing in the NISQ era and beyond, Quantum 2, 79 (2018).

[9] David P. DiVincenzo and Peter W. Shor, Fault-tolerant Error Correction with Efficient Quantum Codes, Phys. Rev. Lett. 77, 3260 (1996).

[10] Earl T. Campbell, Barbara M. Terhal, and Christophe Vuillot, Roads towards fault-tolerant universal quantum computation, Nature 549, 172 (2017).

[11] Jay M. Gambetta, Jerry M. Chow, and Matthias Steffen, Building logical qubits in a superconducting quantum computing system, npj Quantum Information 3, 2 (2017).

[12] Chad Rigetti and Michel Devoret, Fully microwave-tunable universal gates in superconducting qubits with linear couplings and fixed transition frequencies, Phys. Rev. B 81, 134507 (2010).

[13] Jerry M. Chow, A. D. Córcoles, Jay M. Gambetta, Chad Rigetti, B. R. Johnson, John A. Smolin, J. R. Rozen, George A. Keefe, Mary B. Rothwell, Mark B. Ketchen, and M. Steffen, Simple All-Microwave Entangling Gate for FixedFrequency Superconducting Qubits, Phys. Rev. Lett. 107, 080502 (2011).

[14] Sarah Sheldon, Easwar Magesan, Jerry M. Chow, and Jay M. Gambetta, Procedure for systematically tuning up crosstalk in the cross-resonance gate, Phys. Rev. A 93, 060302 (2016).

[15] Susanne Richer, Masters thesis, School: 'Institute of Quantum Information’ RWTH Aachen University, 2013.

[16] J. Rahamim, T. Behrle, M. J. Peterer, A. Patterson, P. A. Spring, T. Tsunoda, R. Manenti, G. Tancredi, and P. J. Leek, Double-sided coaxial circuit QED with out-of-plane wiring, Appl. Phys. Lett. 110, 222602 (2017).

[17] Jay Gambetta, IFF Spring School 2013 Quantum Information Processing Lecture Notes, edited by D. DiVincenzo (Jülich Forschungszentrum, Jülich, 2013), Vol. 339, Chap. B4.

[18] Andrew Patterson, Ph.D. thesis, School: 'Department of Physics' University of Oxford, 2018.

[19] Easwar Magesan and Jay M. Gambetta, Effective Hamiltonian models of the cross-resonance gate, arXiv:1804.04073 (2018).

[20] Michael A. Nielsen and Isaac L. Chuang, Quantum Computation and Quantum Information: 10th Anniversary Edition (Cambridge University Press, Cambridge, 2010).

[21] A. D. Córcoles, Jay M. Gambetta, Jerry M. Chow, John A. Smolin, Matthew Ware, Joel Strand, B. L. T. Plourde, and M. Steffen, Process verification of two-qubit quantum gates by randomized benchmarking, Phys. Rev. A 87, 030301 (2013).

[22] Michael A. Nielsen, A simple formula for the average gate fidelity of a quantum dynamical operation, Phys. Lett. A 303, 249 (2002).

[23] Joseph L. Allen, Robert Kosut, Jaewoo Joo, Peter Leek, and Eran Ginossar, Optimal control of two qubits via a single cavity drive in circuit quantum electrodynamics, Phys. Rev. A 95, 042325 (2017).

[24] Susanna Kirchhoff, Torsten Keßler, Per J. Liebermann, Elie Assémat, Shai Machnes, Felix Motzoi, and Frank K. Wilhelm, Optimized cross-resonance gate for coupled transmon systems, Phys. Rev. A 97, 042348 (2018). 